\title{
The Throughput Analysis of ISA-MAC Algorithm
}

\author{
Song Zu-Xun ${ }^{1}$, Wang Wei-Quan ${ }^{1,2, *}$ and Mao Xiao-Fei ${ }^{1}$ \\ ${ }^{I}$ Northwestern Poly Technical University, Xi'an 710072, China; ${ }^{2}$ Sichuan Jiuzhou Electric Group Co., Ltd. Mianyang \\ Sichuan 621000
}

\begin{abstract}
For AD HOC network designing, disorder transmission which exits in chaos procedure is a challenge how to make sure successful communication without pre-coordinate or back-off-retransmission, this paper base on the ISA-MAC algorithm made by author mentioned in conference and analyze the system throughput, and try to prove its commodity.
\end{abstract}

Keywords: Concatenated coding, disorder transmission, ISA-MAC protocol, throughput analysis.

\section{INTRODUCTION}

ISA-MAC is a kind of chaos channel transmission algorithm, in which transmission signal will collapse for hitting each other due to lacking of pre-ordering or coordination. Traditionally, the technical articles are raised as ALOHA, CSMA previously, which is bad real-time for "back-off" brought by channel collision or technology difficulty by sensing. Later, TDMA raised and used communication-order to improve the successful delivery of probability, but also to accompany the negative effect including time-delay, transmission -sync and extra message cost, so chaos channel transmission is a very important subject, which is a key and provides Ad Hoc system with the capability of real-time, flexible deployment without schedule. ISA-MAC is good at solving the hit problem in chaos channel due to the idea comprising of sub-packet transmission and Concatenated Coding, which is good at reducing hit-probability and correcting damaged-message respectively.

This paper will not focus on the specific of ISA-MAC, and more detail can be referenced in paper [1], but Section 2 will briefly describe the model for theory analysis.

Section 3 will build the mathematical model to analyze the throughput of ISA-MAC algorithm.

Section 4 simulates the theory analysis and draws a conclusion.

\section{IAS-MAC MODEL REVIEW BRIEFLY}

The flow of ISA-MAC protocol as follows:

The flow chart Starts from initialization/idle status, if MAC receives some business information from network layer, this information should be sequenced according to the priority define. If the priority is high, the information will be sent immediately. Otherwise, the information will be pushed into stack and waiting for sending until no higher priority information.
At the moment to send information, system will judge if the information is expired or not. If it is, system will abandon it and pick up the next one to see which one is unexpired. If it is not, system will make a judgment on the basis of channel possession status and will decide the amount of communication traffic that exceeds the channel threshold or not. After all, multi-signal exists and transmits in space at the time, they will interrupt each other and worsen SNR. Even if system provide $\mathrm{FH}-\mathrm{TH}$ and concatenated coding technique to lighten influence, but space channel is limited, the communication can't be executed when the SNR is too low to be accepted and the quality of communication cannot be tolerated either.

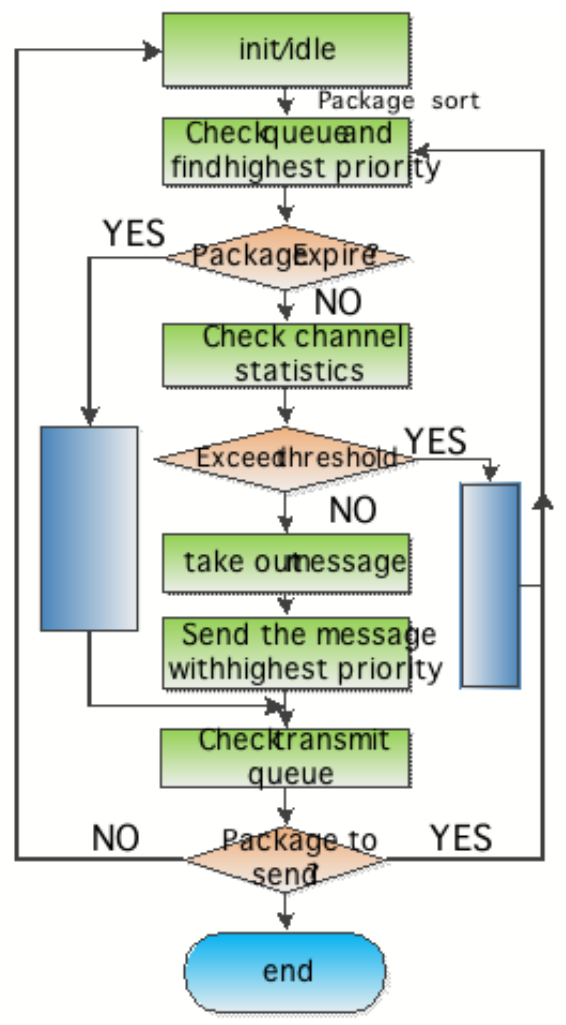

Fig. (1). ISA-MAC protocol flow chart. 
If the possession status is fine, the sub-frame through time jitter will be transmitted at the spot proposed by hopping pattern. If the channel is full, the transmission will be delayed on some algorithm, like binary back off. During back off or before transmission, if there is a new information whose priority is higher than old one, the old one will be pushed into stack and retransmit only if it is still fresh after the new finishing of transition.

After the transmission is over, system check queue will stack and take the next one to send, if the stack is empty, the flow chart will switch to idle and expect new business data arrival.

\section{MATHMETICAL MODEL}

The throughput model supposing the channel noise would be ignored, and $\mathrm{M}$ users exist in system, where design $0 \sim 2^{\wedge} \mathrm{n}$ kinds of messages, 0 presents no message, and $1 \sim 2^{\wedge} \mathrm{n}$ presents the message with length $\mathrm{n}$ respectively. Set $\mathrm{y}$ as the output of channel:

$\left\{\begin{array}{l}y=x_{j} \quad \text { if } x_{i}=0 \text { and } i \neq j \\ y=\text { erasure if more than one } x_{i} \neq 0\end{array}\right.$

P presents the probability of no message, so the probability of transmission of $1 \sim 2^{\wedge} n$ kinds of message would be $(1-p) / 2^{n}$ respectively, under the condition, to make sure the stability of system and build expression as following:

$$
\begin{aligned}
p\left(x_{1} \cdots x_{M} \mathrm{y}\right) & =p_{1}\left(x_{1}\right) \cdots p_{M}\left(x_{M}\right) \mathrm{p}\left(\mathrm{y} / x_{1} \ldots x_{M}\right) \\
& =(1-p) / p \prod_{J=1}^{M} P_{J}=(1-p) p^{M-1}
\end{aligned}
$$

Then, in chaos channel, message would be damaged for overlapping caused by transmission competition, set $1-p$ as the probability of transmission, $p^{2}$ should present the probability of no hitting. So according to (1), induce the amount of information of single user:

$$
T_{i}=(1-p) / p^{2} \prod_{j=1}^{M} p^{2}
$$

And the expression of throughput of system as below:

$T=\sum_{i=1}^{M} T_{i}=M(1-p) p^{2(M-1)}$

Differentiate $\mathrm{p}$, can get the outcome that $\mathrm{T}$ will get max value when $p=1-1 /(2 M-1)$.

$$
\begin{aligned}
T= & {[M /(2 M-1)](1-1 /(2 M-1))^{2(M-1)} } \\
& M \rightarrow \infty, T \rightarrow e^{-1} / 2 .
\end{aligned}
$$

Refer to the coding model mentioned in reference [1], before transmission, the message would be encoded by outercode and will interleave orderly, later, and will be divided into sub-frame with same length finally. The sub-frame would be Encoded with inner- coder either, and will be transmitted.
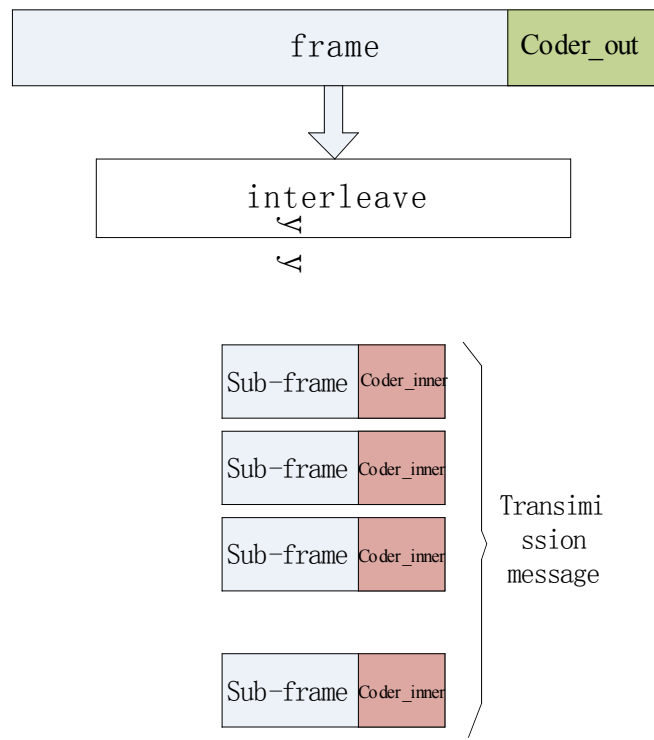

Fig. (2). Concatenated coding.

During transmitting in space, the probability of collision is so high without ordering or coordinating in advance. If collision happens and sub-frame is damaged, the system can use inner-code to correct error and recover the subinformation, if not to abandon the sub- frame. If sub-frame is damaged so hard and can't be fixed by inner-code, the system still can use the error correction of outer-code to overcome the error and recover the original information, which improves the ability of transmission.

If some user has a message to send, set $1-p$ as the probability of transmission during the period decided by the length of message and the duration of single bit, so $p^{2(M-1)}$ should be the probability of avoiding, $1-p^{M-1}$ should be the probability of losing respectively, reasonably. In case that sub-frame could be recovered in the condition that the damage rate is less than $f$, the probability of recovery as following:

$$
\begin{aligned}
& {\left[p\left(\mathrm{D}_{j} \leq f\right) p(a \text { message take two durations, and }\right.} \\
& p_{\text {cover }}=\prod_{j=1}^{M} p\left(\mathrm{D}_{j}>f\right) p(a \text { message take one duration, and } \\
& \text { no overlap with anotherfone } \\
& =\left[f p^{2}+(1-f) \mathrm{p}\right]^{M-1}
\end{aligned}
$$

$\mathrm{D}_{j}$ Present the difference of launch time between users.

$$
\begin{gathered}
\text { So, } \\
\begin{aligned}
p_{\text {cover }} & =-d p_{\text {cover }} / d f \\
& =(\mathrm{M}-1) /(1-p) p^{M-1}(1-f(1-p))^{M-2}
\end{aligned}
\end{gathered}
$$

The mean of the probability of recovery could be deduced

$$
\begin{aligned}
& \int_{0}^{1} p(f) f d f+1 \cdot p^{2(\mathrm{M}-1)} \\
& =\left(1-p^{M}\right) p^{M-1} /[(\mathrm{M}-1)(1-p)]
\end{aligned}
$$




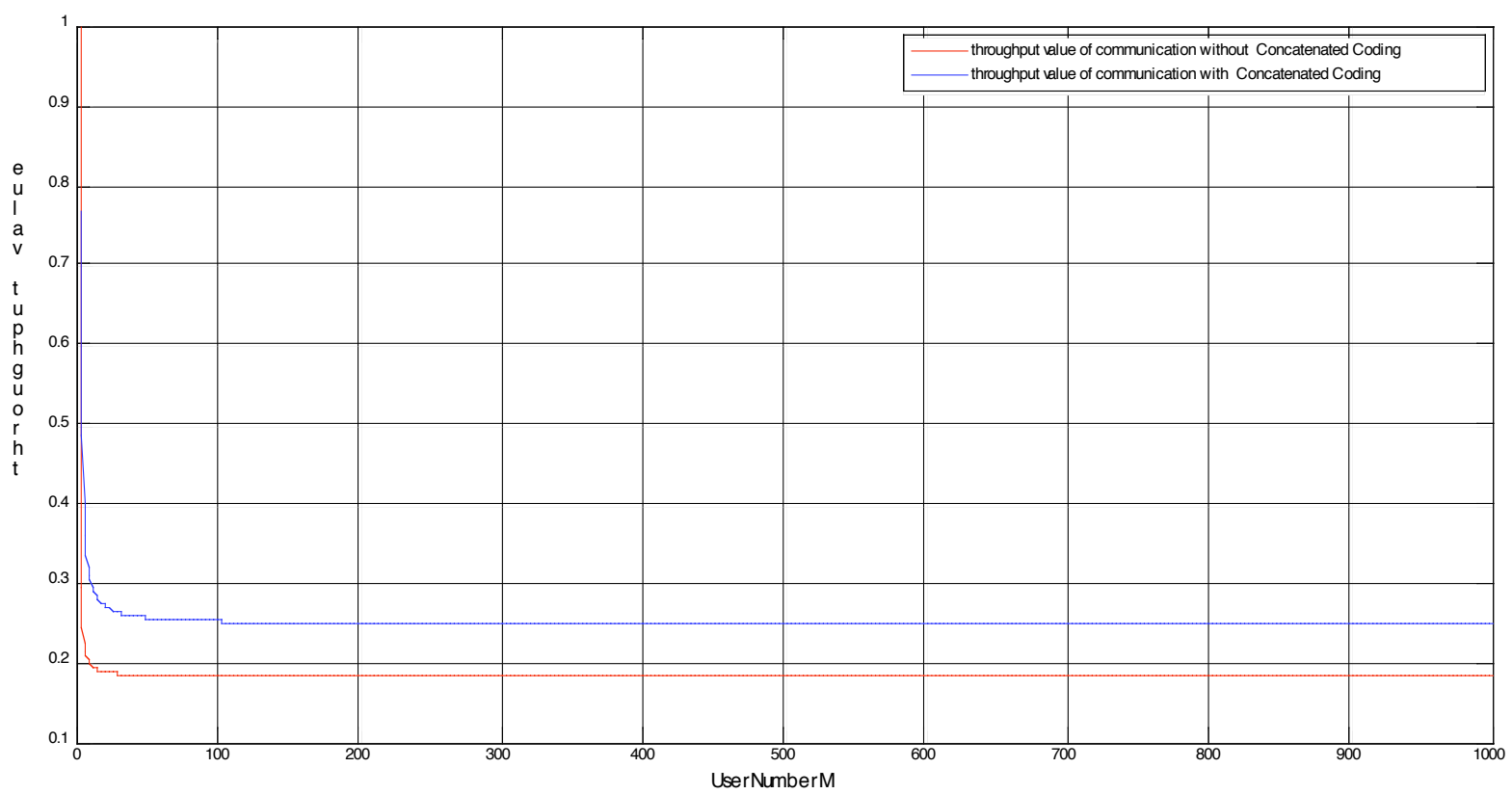

Fig. (3). Throughput value comparison.

For M users, the throughput would be:

$$
T=M\left(1-p^{M}\right) p^{M-1} /(\mathrm{M}-1)
$$

when $p=[(\mathrm{M}-1) /(2 \mathrm{M}-1)]^{1 / M}, T$ get max value:

$$
\begin{aligned}
T= & {[M /(M-1)][M /(2 M-1)][(M-1) /(2 M-1)]^{(M-1) / M} } \\
& \mathrm{M} \rightarrow \infty, \mathrm{T} \rightarrow 1 / 4 .
\end{aligned}
$$

\section{SIMULATION AND CONCLUSION}

In a word, the equation (4) presents the throughput value of communication without Concatenated Coding, and trend to $e^{-1} / 2$, and the equation (10) present in the throughput value of communication with Concatenated Coding, and trends to $1 / 4$. Obviously, Concatenated Code works well on improving the throughput of the system. Use the ability of correcting of code, system can survive the chaos hitting and noise problem, and can transmit at any time

\section{CONFLICT OF INTEREST}

The authors confirm that this article content has no conflict of interest.

\section{ACKNOWLEDGEMENTS}

Declared none.

\section{REFERENCES}

[1] W.W. Quan, S.Z. Xun and M. Fei, "A protocol of ISA-MAC using FH-TH associate with concatenated coding," Appl. Mech. Mater., vol. 602-605, pp. 3721-3726, 2014

[2] W.S. Wong, "New protocol sequences for random access channels without feedback," IEEE Trans. Inform., vol. 53, no. 6, pp. 20602071, jun. 2007

[3] V.I. Levenshtein, "Conflict-avoiding codes and cyclic triple systems, "Probl. Inform. Transmis., vol. 43, no. 3. pp. 199-212, 2007.

[4] M. Mishima, H.L. Fu, and S. Uruno, "Optimal conflict-avoiding codes of length $\mathrm{n} \equiv 0(\bmod 16)$ and weight 3 ," Design, codes and Cryptography, vol. 52, pp. 275-291, 2009.

[5] Y. Zhang, K.W. Shum and W.S. Wong, "Strongly conflict-avoiding codes," SIAM J. on Discrete Math, vol. 25, no. 3, pp. 1035-1053, July 2011.

[6] G.D. Forney "Concatenated Codes" MIT research monograph No. 37 The MIT Press Cambridge Mass. 1966.

[7] G.W. Goodman Jr, "Machine-to-machine," Aviation Week and Space Technology (New York), vol. 165, no. 12, pp. 40-44, Sep. 2006

[8] G. Bigalk, T. Rogge, "Developing technologies in the US Navy super hornet," In: Society of Flights Test Engineers, SFTE 36th Annual Symposium Proceedings, pp. 81-98, 2005.

[9] W.B. Scott, "TAC-air Internet," Aviation Week Space Technology (New York), vol. 164, no. 25, pp. 51-53, June 19, 2006

[10] A.M. Lewis, "Quality of service for tactical data links: TDMA with dynamic scheduling," In: Proceedings - IEEE Military Communications Conference MILCOM, vol. 2005. 